ISSN 0103-9954

\title{
BIOMASSA E NUTRIENTES NO CORTE RASO DE UM POVOAMENTO DE Pinus taeda L. DE 27 ANOS DE IDADE EM CAMBARÁ DO SUL - RS
}

\author{
BIOMASS AND NUTRIENTS IN A 27 YEARS Pinus taeda L. CLEAR CUTTING STAND IN \\ CAMBARÁ DO SUL, RIO GRANDE DO SUL STATE
}

\author{
Mauro Valdir Schumacher ${ }^{1}$ Rudi Witschoreck ${ }^{2}$ Francine Neves Calil ${ }^{3}$ Vicente Guilheme Lopes ${ }^{4}$
}

\begin{abstract}
RESUMO
Este trabalho, realizado em um povoamento de Pinus taeda de 27 anos de idade no município de Cambará do Sul (RS) teve como objetivos: estimar a produção de biomassa, o estoque de nutrientes e avaliar o impacto nutricional de diferentes intensidades de remoção de biomassa com a colheita florestal. A biomassa foi estimada por meio do ajuste de equações de regressão, com coleta de 15 árvores distribuídas em 5 classes diamétricas. O estoque de nutrientes foi obtido pelo produto entre o conteúdo médio de nutrientes em cada componente da biomassa e o número de árvores por classe diamétrica por hectare. A biomassa de Pinus taeda, acima do solo, foi estimada em 266,08 $\mathrm{Mg} \mathrm{ha}^{-1}$, sendo composta por: $69,1 \%$ de madeira do tronco, $17,1 \%$ de galhos vivos, $6,7 \%$ de casca do tronco, $3,8 \%$ de galhos secos e $3,4 \%$ de acículas. O estoque de nutrientes na biomassa, em $\mathrm{kg} \mathrm{ha}^{-1}$, foi estimado em: 511,96 de N; 44,39 de P; 174,27 de K; 310,77 de Ca; 103,80 de Mg; 115,36 de S; 2,94 de B; 0,62 de Cu; 17,34 de Fe; 36,70 de Mn e 4,46 de Zn. A distribuição relativa do estoque total de nutrientes nos componentes da biomassa de Pinus taeda apresentou a seguinte sequência: madeira do tronco (43,6\%), galhos vivos $(24,8 \%)$, acículas $(19,0 \%)$, casca do tronco $(8,7 \%)$ e galhos secos $(3,9 \%)$. A colheita de toda biomassa acima do solo, quando comparada com a retirada apenas da madeira do tronco, acarreta uma elevação na exportação de nutrientes que pode variar de $58,0 \%$ a $127,4 \%$, dependendo do nutriente, enquanto a remoção de biomassa aumentou 40,8 \%.
\end{abstract}

Palavras-chave: colheita florestal; produtividade; ciclagem de nutrientes; sustentabilidade.

\section{ABSTRACT}

This study was conducted in a 27 year Pinus taeda stand in Cambará do Sul, Rio Grande do Sul state and aimed to estimate the biomass production, nutrient stock and to evaluate the nutritional impact in different forest harvesting intensities. Biomass was estimated through regression equation adjustments, with the cut of 15 trees distributed in 5 diametric classes. Nutrients stock was obtained through the product between the average content of nutrients in each biomass component and the number of trees per diametric class per hectare. Pinus taeda above ground biomass was estimated in $266.08 \mathrm{Mg} \mathrm{ha}^{-1}$, being $69.1 \%$ of wood, 17.1 of live branches, $6.7 \%$ of bark, $3.8 \%$ of dead branches and $3.4 \%$ of needles. Nutrients stock in biomass $\left(\mathrm{kg} \mathrm{ha}^{-1}\right)$ was estimated in: 511.96 of N, 44.39 of $\mathrm{P}, 174.27$ of K, 310.77 of $\mathrm{Ca}, 103.80$ of $\mathrm{Mg}, 115.36$ of S, 2.94 of B, 0.62 of $\mathrm{Cu}, 17.34$ of Fe, 36.70 of $\mathrm{Mn}$ and 4.46 of $\mathrm{Zn}$. Nutrients stock relative distribution in Pinus taeda biomass components showed the following sequence: wood (43.6\%), live branches $(24.8 \%)$, needles $(19.0 \%)$, bark $(8.7 \%)$ and dead branches $(3.9 \%)$. Total above ground biomass harvest, when compared to

1. Engenheiro Florestal, Dr., Professor Associado do Departamento de Ciências Florestais, Universidade Federal de Santa Maria, Cidade Universitária, Av. Roraima 1000, CEP 97105-900, Santa Maria (RS). Bolsista do CNPq. schumacher@pq.cnpq.br

2. Engenheiro Florestal, Doutorando do Programa de Pós-graduação em Engenharia Florestal, Centro de Ciências Rurais, Universidade Federal de Santa Maria, Cidade Universitária, CEP 97105-900, Santa Maria (RS). Bolsista do CNPq. rwitschoreck@yahoo.com.br

3. Engenheira Florestal, Dra ${ }^{\text {. }}$, Professora da Escola de Agronomia e Engenharia de Alimentos da Universidade Federal de Goiás, Rodovia Goiânia, Nova Veneza, km 0, Campus Samambaia, CEP 74690-900, Goiânia (GO). francine.cali1@terra.com.br

4. Engenheiro Florestal, Doutorando do Programa de Pós-graduação em Engenharia Florestal, Centro de Ciências Rurais, Universidade Federal de Santa Maria, Cidade Universitária, CEP 97105-900, Santa Maria (RS). Bolsista do CNPq.viguilopes@yahoo.com.br

Recebido para publicação em 23/06/2010 e aceito em 11/04/2012 
only wood removal, leads to nutrients export increase that can vary from $58.0 \%$ to $127.4 \%$, depending on the chemical element, while biomass removal increases $40.8 \%$.

Keywords: forest harvesting; productivity; nutrient cycling; sustainability.

\section{INTRODUÇÃO}

Os povoamentos florestais são sistemas abertos sujeitos a um grande número de entradas e saídas de elementos químicos, muitos deles reconhecidos como nutrientes essenciais ao desenvolvimento vegetal (EPSTEIN e BLOOM, 2006; PALLARDY, 2008).

$\mathrm{O}$ entendimento da ciclagem de nutrientes nos ecossistemas florestais é um pré-requisito essencial para a compreensão e a predição dos efeitos da nutrição no crescimento da floresta (LANDSBERG, 1986); e um dos aspectos fundamentais para a manutenção da produtividade florestal (REIS e BARROS, 1990).

A ciclagem de nutrientes em ecossistemas florestais pode ser dividida em dois ciclos principais, segundo Ramezov (1959): o geoquímico, que envolve os processos de entrada e saída de nutrientes no ecossistema, e o biológico, que trata das transferências no interior do ecossistema. Para este último, Switzer e Nelson (1972), sugeriram uma divisão em ciclo biogeoquímico, para transferências entre o solo e a planta, e bioquímico, para o processo de translocação dentro da planta.

As entradas de nutrientes no sistema podem ocorrer através das deposições atmosféricas (secas e úmidas), intemperismo geológico, fixação biológica de nitrogênio e fertilização, enquanto que as saídas incluem a volatilização pelas queimadas ou pela desnitrificação, lixiviação e erosão hídrica, assim como, a colheita da biomassa (PRITCHETT, 1990). Normalmente a colheita florestal é a principal via de exportação de nutrientes dos sítios florestais, sobretudo, se realizada em rotações curtas e envolvendo outros componentes da biomassa, além da madeira.

A deposição de nutriente via atmosfera depende de fatores naturais e antrópicos (WARING e SCHLESINGER, 1985), com grande variação de acordo com a localização geográfica, estação do ano e proximidade a grandes centros urbanos, industriais e agrícolas (PRINTCHETT, 1990).

Além da colheita florestal, toda prática de manejo que intensifica o grau e o período de exposição do solo sem a proteção da vegetação ou de resíduos orgânicos, provoca aumento de perdas do sistema. Neste contexto, o uso da queima para elimi- nação de resíduos de colheita é uma prática nociva que acarreta perdas de nutrientes por volatilização, lixiviação e erosão, principalmente quando associado a altos índices pluviométricos e condições topográficas desfavoráveis (PRITCHETT, 1990; LANDSBERG, 1986).

Em muitas partes do mundo, o manejo inadequado da biomassa e seus resíduos têm sido a principal causa da redução da produtividade dos solos florestais. A biomassa é fonte de nutrientes, sendo em muitos casos o principal compartimento de armazenamento, como é o caso de muitas áreas de floresta tropical (PRITCHETT, 1990).

Desse modo, o conhecimento da distribuição da biomassa e do estoque de nutrientes nos diferentes componentes e nas distintas fases de desenvolvimento dos povoamentos florestais pode subsidiar inúmeras práticas que visam à manutenção da produtividade dos sítios florestais, tais como: definição da idade e intensidade de colheita, manejo dos resíduos florestais, adubação de reposição, preparo de solo, entre outras.

Portanto, o presente trabalho teve como objetivos: estimar a produção de biomassa, o estoque de nutrientes e avaliar o impacto nutricional de diferentes intensidades de remoção de biomassa com a colheita florestal.

\section{MATERIAL E MÉTODO}

\section{Localização e caracterização da área do estudo}

$\mathrm{O}$ estudo foi realizado em um povoamento de Pinus taeda, com 27 anos de idade, pertencente à empresa Reflorestadores Unidos S.A., localizado no município de Cambará do Sul, Rio Grande do Sul, coordenadas $29^{\circ} 13^{\prime} 34^{\prime \prime}$ de latitude sul e $50^{\circ} 18^{\prime} 23^{\prime \prime}$ de longitude oeste e a uma altitude de $1.030 \mathrm{~m}$.

Segundo a classificação de Köppen, o tipo fundamental do clima da região é o Cfb (MORENO, 1961), com uma precipitação anual média de $1.906 \mathrm{~mm}$, registrada no período de 1997 - 2007 (INSTITUTO NACIONALDE METEOROLOGIA, 2008). A temperatura média nesse período foi de $15,5^{\circ} \mathrm{C}$, com valores médios de $20,9^{\circ} \mathrm{C}$ para as máximas e $10,9^{\circ} \mathrm{C}$ para as mínimas, com um extremo para as temperaturas mínimas absolutas de $-7,2{ }^{\circ} \mathrm{C}$. Na região ocorrem cerca de 40 geadas por ano, prin- 
cipalmente no período de abril a novembro e queda de neve em cerca de três dias por ano (MORENO, 1961).

Quanto aos solos, os materiais de origem são rochas basálticas, resultantes do derrame basáltico do Triássico Superior, predominando solos rasos com horizonte A de coloração escura, com baixa saturação de bases e teores elevados de alumínio trocável. O solo da região é do tipo Cambissolo Húmico Alumínico (STRECK et al., 2008).

\section{Amostragem e estimativa da biomassa}

O inventário florestal da área de estudo foi realizado a partir de amostragem aleatória, distribuindo-se cinco parcelas de $600 \mathrm{~m}^{2}$ de área, onde foram medidos, todos os diâmetros a altura do peito (DAP), com auxílio de uma suta, e a altura total de $20 \%$ das árvores com o uso de um hipsômetro de precisão. O restante das alturas foi estimado por meio de equação de regressão com base na relação hipsométrica. A partir das informações coletadas, estabeleceu-se a distribuição diamétrica do povoamento com a divisão de cinco classes de diâmetro, com amplitude de $7 \mathrm{~cm}$. Em cada uma dessas classes foram abatidas 3 árvores, totalizando 15 árvores abatidas. Após abate de cada árvore-amostra, a biomassa foi triada, separando-se nos seguintes componentes: acículas, galhos vivos, galhos secos, madeira do tronco e casca do tronco. Em seguida, cada um dos componentes foi pesado para quantificação da biomassa úmida e, após isso, separou-se uma amostra representativa de cada componente. Para determinação do teor de umidade e análise química, casca e madeira tiveram três amostras coletadas em três posições ao longo do tronco (DAP - 1,3 m de altura, $50 \%$ da altura total e no ponto médio da ponteira determinada com base no diâmetro mínimo aproveitável de $10 \mathrm{~cm}$ ), com propósito de aumentar a representatividade das amostras e cobrir possíveis variações, principalmente na concentração de nutrientes.

No Laboratório de Ecologia Florestal do Departamento de Ciências Florestais/UFSM, as amostras coletadas foram secas em estufa com circulação e renovação de ar a temperatura de $70^{\circ} \mathrm{C}$, para determinação do teor de umidade.

A modelagem de regressão seguiu o procedimento "forword" (proc stepwise e opção forward) do pacote SAS (1993), com verificação da normalidade da distribuição dos resíduos pelo teste de Shapiro-Wilk, homogeneidade da variância pelo teste de White e independência dos resíduos pela estatística de Durbin-Watson (SCHNEIDER et al., 2009).

A estimativa da biomassa foi realizada mediante ajuste de equações de regressão, tendo como variáveis dependentes a biomassa: acículas, galhos vivos, galhos secos, casca do tronco e madeira do tronco e biomassa total acima do solo, em $\mathrm{kg}$ por árvore-amostra, e as seguintes variáveis independentes: d (diâmetro a altura do peito), h (altura total), bem como, transformações aritméticas e logarítmicas das variáveis. Depois da seleção da melhor equação de regressão, para cada componente, esta foi aplicada aos dados do inventário florestal para estimar a biomassa de cada componente das árvores-amostra.

\section{Estimativa dos nutrientes e implicações da colheita florestal}

Após secagem, as amostras foram moídas em moinho de laminas do tipo Wiley com peneira de 20 mesh. As determinações analíticas foram realizadas no Laboratório de Ecologia Florestal do Departamento de Ciências Florestais/UFSM, segundo Miyazawa et al. (1999). O N foi determinado pelo método Kjeldhal, no extrato de digestão sulfúrica; B por espectrofotometria com digestão seca; e os demais elementos, no extrato de digestão nítrico-perclórico, sendo $\mathrm{Ca}, \mathrm{Mg}, \mathrm{Cu}, \mathrm{Fe}, \mathrm{Mn}$ e $\mathrm{Zn}$ por espectrofotometria de absorção atômica, $\mathrm{P}$ por espectrofotometria, K por fotometria de chama e $\mathrm{S}$ por turbidimetria.

$\mathrm{O}$ estoque de nutriente por unidade de área foi obtido através do produto entre o conteúdo médio de nutrientes em cada componente da biomassa e o número de árvores por classe diamétrica por hectare.

A fim de avaliar a taxa de conversão de nutrientes em biomassa e consequentemente, a exportação relativa de nutrientes por unidade de biomassa de cada componente das árvores, foi estimado o coeficiente de utilização biológico (CUB), obtido pelo quociente entre a biomassa e a quantidade de nutriente, ambos com a mesma unidade (BARROS et al., 1986).

O impacto nutricional da colheita florestal foi avaliado com base na exportação de nutrientes mediante possíveis intensidades de utilização da biomassa: remoção apenas da madeira do tronco (situação ideal do ponto de vista da sustentabilidade nutricional); remoção de outros componentes das árvores, além da madeira; e a remoção de toda a biomassa acima do solo. 


\section{RESULTADOS E DISCUSSÃO}

\section{Estoque de biomassa}

$\mathrm{Na}$ Tabela 1 são apresentadas as equações de regressão utilizadas para estimar a biomassa de cada componente das árvores de Pinus taeda. Em geral, as equações apresentaram-se altamente significativas e com elevados valores para as estatísticas que medem o grau de ajuste dos modelos, como o coeficiente de determinação e o erro padrão da estimativa. Apenas a equação 3 , ajustada para galhos secos, apresentou baixo valor para $\mathrm{R}^{2}$ (53\%) e alto valor para Sxy (43\%). Em função da maior variabilidade, geralmente, a estimativa da biomassa de galhos secos apresenta um maior erro associado, mas, em virtude da pequena representatividade desse componente, em termos relativos, não traz maiores problemas para a estimativa da biomassa total (SCHUMACHER et al., 2002; SCHUMACHER et al., 2003; WITSCHORECK, 2008; CALDATO, 2011).

Os pressupostos de normalidade, homogeneidade e independência dos resíduos, foram atendidos, como se verifica, respectivamente, pelos testes de Shapiro-Wilk, White e Durbin-Watson (Tabela 1). Com base nos valores de probabilidade $(>0,05)$ associados aos testes de Shapiro-Wilk e de White, é possível afirmar que os resíduos apresentam distribuição normal e têm a mesma variância nos diferentes níveis dos previsores (homocedásticos). A estatística de Durbin-Watson, com variação entre 0 e 4, indica se os resíduos estão correlacionados em série (dependentes), onde escores menores que 1 e maiores que 3 são tidos como problemáticos, enquanto que 2 é o valor ideal.

A alocação de biomassa nos componentes das árvores de Pinus taeda apresentou a seguinte sequência de acúmulo: madeira do tronco $(69,1 \%)$; galhos vivos $(17,1 \%)$; casca do tronco $(6,7 \%)$; acículas $(3,4 \%)$ e galhos secos $(3,8 \%)$ (Tabela 2). Trata-se de uma distribuição de biomassa típica de povoamentos adultos, que se caracterizam pelo maior acúmulo de biomassa no tronco (75,8 \%) (REIS e BARROS, 1990; MILLER, 1995; GONÇALVES et al., 2004).

Bizon (2005), ao estudar a sustentabilidade de produção com base no balanço de nutrientes em 8 povoamentos de Pinus taeda, com idades en-

TABELA 1: Equações de regressão utilizadas na estimativa da biomassa total e dos diferentes componentes das árvores de Pinus taeda de 27 anos de idade, em Cambará do Sul, RS.

TABLE 1: Regression equations used for total biomass and biomass components estimation from 27 year Pinus taeda trees, in Cambará do Sul, RS state.

\begin{tabular}{lll}
\hline & Componente & Equações \\
\hline 1 & Acículas & $\operatorname{lny}=-4,055687+0,931219 \cdot \ln \left(\mathrm{h}^{-1} \cdot \mathrm{d}^{3}\right)$ \\
2 & Galhos vivos & $\operatorname{lny}=-1,852750+1,610828 \cdot \ln \left(\mathrm{h}^{-1} \cdot \mathrm{d}^{2}\right)$ \\
3 & Galhos secos & $\mathrm{y}=7,694635+0,008984 \cdot\left(\mathrm{h}^{-1} \cdot \mathrm{d}^{3}\right)$ \\
4 & Casca do tronco & $\operatorname{lny}=3,578384+0,000337 \cdot \mathrm{d}^{2}$ \\
5 & Madeira do tronco & $\operatorname{lny}=-1,608288+0,558150 \cdot \ln \left(\mathrm{d}^{3} \cdot \mathrm{h}\right)$ \\
6 & Biomassa total & $\operatorname{lny}=-0,513258+1,966150 \cdot \ln \mathrm{l}$ \\
\hline
\end{tabular}
Estatísticas de ajuste e condicionantes das regressões

\begin{tabular}{|c|c|c|c|c|c|c|c|c|c|c|}
\hline & \multirow{2}{*}{ Prob. $>$ F } & \multirow{2}{*}{$\mathrm{R}^{2}$} & \multirow{2}{*}{ Syx } & \multirow{2}{*}{ Syx (\%) } & \multicolumn{2}{|c|}{ Shapiro-Wilk } & \multicolumn{2}{|c|}{ White } & \multicolumn{2}{|c|}{ D. Watson } \\
\hline & & & & & Valor & Prob. & Valor & Prob. & Valor & $\alpha$ \\
\hline 1 & 0,000 & 0,9133 & 0,1748 & 4,82 & 0,95 & 0,444 & 1,25 & 0,536 & 2,60 & 0,01 \\
\hline 2 & 0,000 & 0,9225 & 0,1886 & 3,54 & 0,90 & 0,084 & 1,75 & 0,418 & 2,16 & 0,01 \\
\hline 3 & 0,002 & 0,5324 & 21,9909 & 43,26 & 0,95 & 0,522 & 1,20 & 0,549 & 1,33 & 0,05 \\
\hline 4 & 0,000 & 0,8098 & 0,1595 & 3,60 & 0,97 & 0,822 & 0,86 & 0,652 & 1,88 & 0,01 \\
\hline 5 & 0,000 & 0,9633 & 0,0709 & 1,05 & 0,96 & 0,652 & 0,77 & 0,682 & 2,22 & 0,01 \\
\hline 6 & 0,000 & 0,9793 & 0,0596 & 0,84 & 0,92 & 0,198 & 1,75 & 0,417 & 2,59 & 0,01 \\
\hline
\end{tabular}

Em que: $\ln =$ logaritmo natural; $\mathrm{y}=$ biomassa em $\mathrm{kg}$ do respectivo componente; $\mathrm{d}=$ DAP (diâmetro a altura do peito, em $\mathrm{cm}) ; \mathrm{h}=$ altura total, em m; Prob. = nível de significância para a estatística; $\mathrm{R}^{2}=$ coeficiente de determinação; Syx $=$ erro padrão da estimativa absoluto; Syx $(\%)=$ erro padrão da estimativa relativo; $\alpha=$ nível de probabilidade associado ao teste. 
TABELA 2: Variáveis dendrométricas e biomassa $\left(\mathrm{Mg} \mathrm{ha}^{-1}\right)$ nos diferentes componentes das árvores de Pinus taeda de 27 anos de idade, em Cambará do Sul, RS.

TABLE 2: Dendrometric variables and biomass $\left(\mathrm{Mg} \mathrm{ha} \mathrm{h}^{-1}\right)$ in different components from 27 year Pinus taeda trees, in Cambará do Sul, RS state.

\begin{tabular}{cc}
\hline Variável & Estimativa \\
\hline DAP $(\mathrm{cm})$ & 48,9 \\
Altura $(\mathrm{m})$ & 27,4 \\
Número de árvores por hectare & 243 \\
Volume do tronco com casca $\left(\mathrm{m}^{3} \mathrm{ha}^{-1}\right)$ & 643,70 \\
Volume do tronco sem casca $\left(\mathrm{m}^{3} \mathrm{ha}^{-1}\right)$ & 550,73 \\
\hline Acículas & 9,04 \\
Galhos vivos & 45,44 \\
Galhos secos & 10,00 \\
Madeira do tronco & 183,83 \\
Casca do tronco & 17,77 \\
Biomassa total & 266,08 \\
\hline
\end{tabular}

tre 19 e 29 anos e número de árvores por hectare de 125 a 1.300 , verificou uma partição de biomassa, considerando-se apenas os componentes acima do solo, de 77,6 \% de madeira do tronco, $12,1 \%$ de galhos, 7,0 \% de casca do tronco e 3,3\% de acículas.

Barros Filho (2003), avaliando a distribuição de biomassa em plantios de Pinus taeda de 2, 4 e 6 anos de idade, estimou a biomassa de tronco (casca e madeira) em 54,7\%, $57 \%$ e $62,2 \%$, ao passo que a biomassa de acículas foi de $31 \%$, $20,9 \%$ e $16,8 \%$, respectivamente; mostrando uma nítida inversão na prioridade de alocação de fotoassimilados com a idade dos povoamentos.

A sequência de alocação de biomassa coincide com os resultados obtidos por Witschoreck (2008), para um povoamento de Pinus taeda de 17 anos de idade, na mesma região deste estudo, com exceção das acículas (5,6 \%), que apresentaram valor superior em relação aos galhos $\operatorname{secos}(3,3 \%)$. Ainda em relação ao mesmo autor, além das acículas, outro valor divergente diz respeito ao reduzido percentual de galhos verdes $(10,3 \%)$. Esses resultados devem estar relacionados com a maior densidade de plantas por hectare (581 árvores), apesar da menor idade do povoamento. Ladeira et al. (2001) e Leite et al. (1997), verificaram, em espécies de eucalipto, que à medi- da que a densidade de plantas aumenta, a biomassa total por unidade de área também aumenta, ao passo que a produção de biomassa total por indivíduo diminui, mas com elevação da biomassa relativa de tronco.

Também em Cambará do Sul, Schumacher et al. (2006), avaliando a exportação de nutrientes e biomassa no primeiro desbaste de um povoamento de Pinus taeda de 9 anos de idade com densidade de plantio de 1.666 plantas por hectare, verificaram a seguinte distribuição de biomassa: madeira do tronco (70 \%); galhos vivos (10,5\%); casca do tronco $(9,6 \%)$; acículas $(8,2 \%)$ e galhos $\operatorname{secos}(1,6 \%)$.

Baldwin Jr. et al. (2000), ao avaliarem o efeito do espaçamento entre plantas e de desbastes em Pinus taeda, aos 38 anos de idade, constataram que os povoamentos mais intensamente desbastados apresentaram alteração no padrão de distribuição de biomassa, principalmente a alocação de mais biomassa para galhos e folhas.

Em relação ao efeito da idade na distribuição de biomassa, Valeri (1988), ao avaliar a exportação de biomassa em povoamentos de Pinus taeda, por ocasião dos desbastes, verificou entre povoamentos de 7 e 14 anos de idade, diminuição da biomassa relativa de acículas, galhos e casca, respectivamente: de $13 \%$ para $5,1 \%$, de $23 \%$ para $15,3 \%$ e de $7,4 \%$ para $6,2 \%$; enquanto a biomassa de madeira aumentou de $56,6 \%$ para $73,4 \%$. A mesma tendência também foi verificada por outros autores em povoamentos de Eucalyptus grandis e de Pinus taeda (PEREIRA et al., 1984; ZHANG et al., 2004; MARTIN e JOKELA, 2004).

Bizon (2005), quando estudou 8 povoamentos de Pinus taeda, atribuiu aos diferentes níveis de produtividade, a diferença na alocação de biomassa para o componente raiz.

Práticas intensivas de manejo em povoamentos de Pinus taeda, tais como controle de plantas invasoras, irrigação, fertilização, dependendo dos níveis de fertilidade e déficit hídrico do sítio, podem provocar alterações na alocação de biomassa nos diferentes componentes das árvores (ALBAUGH et al., 2004; MARTIN e JOKELA, 2004).

\section{Estoque de nutrientes}

Na Tabela 3 é possível verificar o conteúdo de macro e micronutrientes nos diferentes componentes da biomassa de Pinus taeda aos 27 anos de idade. 
TABELA 3: Estoque de nutrientes $\left(\mathrm{kg} \mathrm{ha}^{-1}\right)$ nos diferentes componentes da biomassa de Pinus taeda de 27 anos de idade, em Cambará do Sul, RS.

TABLE 3: Nutrients stock $\left(\mathrm{kg} \mathrm{ha}^{-1}\right)$ in different components from 27 year Pinus taeda trees, in Cambará do Sul, RS state.

\begin{tabular}{ccccccc}
\hline \multirow{2}{*}{ Nutriente } & \multicolumn{5}{c}{ Componente da biomassa } \\
\cline { 2 - 7 } & Acículas & Galhos vivos & Galhos secos & Casca do tronco & Madeira do tronco & Total (\%) \\
\hline $\mathrm{N}$ & 140,65 & 108,92 & 16,54 & 47,09 & 198,76 & 38,7 \\
$\mathrm{P}$ & 11,26 & 10,87 & 0,91 & 3,42 & 17,93 & 3,4 \\
$\mathrm{~K}$ & 41,14 & 46,85 & 1,23 & 13,4 & 71,65 & 13,2 \\
$\mathrm{Ca}$ & 30,52 & 93,11 & 21,59 & 32,33 & 133,22 & 23,5 \\
$\mathrm{Mg}$ & 11,37 & 29,88 & 3,65 & 7,43 & 51,47 & 7,9 \\
$\mathrm{~S}$ & 8,72 & 24,87 & 4,37 & 8,04 & 69,36 & 8,7 \\
\hline $\mathrm{B}$ & 0,27 & 0,72 & 0,11 & 0,24 & 1,59 & 0,2 \\
$\mathrm{Cu}$ & 0,05 & 0,16 & 0,02 & 0,06 & 0,33 & $<0,1$ \\
$\mathrm{Fe}$ & 1,37 & 3,53 & 0,68 & 1,25 & 10,52 & 1,3 \\
$\mathrm{Mn}$ & 5,27 & 8,55 & 2,02 & 0,91 & 19,94 & 2,8 \\
$\mathrm{Zn}$ & 0,29 & 1,08 & 0,20 & 0,34 & 2,55 & 0,3 \\
Total (\%) & $(19,0)$ & $(24,8)$ & $(3,9)$ & $(8,7)$ & $(43,6)$ & 100,0 \\
\hline
\end{tabular}

Em que: Valores entre parênteses referem-se à contribuição relativa do respectivo componente da biomassa ou nutriente, em relação ao estoque total de nutrientes.

Considerando-se a biomassa total, o estoque de nutrientes obedece a seguinte ordem de acúmulo: $\mathrm{N}>\mathrm{Ca}>\mathrm{K}>\mathrm{S}>\mathrm{Mg}>\mathrm{P}>\mathrm{Mn}>\mathrm{Fe}>\mathrm{Zn}$ $>\mathrm{B}>\mathrm{Cu}$; ao passo que, considerando o estoque de nutrientes nos diferentes componentes, tem-se a sequência: madeira do tronco $>$ galhos verdes $>$ acículas $>$ casca do tronco $>$ galhos secos.

A sequência de acúmulo observada neste trabalho, para os três nutrientes mais abundantes: $\mathrm{N}>\mathrm{Ca}>\mathrm{K}$, coincide com os apresentados por Witschoreck (2008) e Caldato (2011), para povoamentos de Pinus taeda, respectivamente, com 17 e 13 anos de idade.

Enquanto algumas espécies de Eucalyptus são notórias pelo elevado acúmulo de $\mathrm{Ca}$ na biomassa, sobretudo na casca, no Pinus taeda, independentemente da fase de desenvolvimento do povoamento, o $\mathrm{N}$ é o elemento em maior quantidade (VALERI, 1988; OKI, 2002; BARROSO FILHO, 2003; BIZON, 2005; VOIGTLAENDER, 2007; WITSCHORECK, 2008; CALDATO, 2011).

A sequência $\mathrm{Ca}>\mathrm{K}$, como segundo e terceiro nutrientes mais abundantes, parece estabilizar em povoamentos em torno dos 10 anos de idade, pelo que indica alguns estudos (VALERI, 1988; BARROS FILHO, 2003; VOIGTLAENDER, 2007). Essa tendência pode ser verificada nos resul- tados obtidos por Valeri (1988) em povoamentos de Pinus taeda de 7, 10 e 14 anos de idade, onde o estoque de $\mathrm{Ke} \mathrm{Ca}$ apresentaram os seguintes valores (em $\left.\mathrm{kg} \mathrm{ha}^{-1}\right): 114,9$ e 88,$7 ; 144,0$ e 152,2 e 120,7 e 190,9, respectivamente. O comportamento desses dois nutrientes em função da idade está ligado à diferença de mobilidade no tecido vegetal. Enquanto o $\mathrm{Ca}$, na grande maioria, permanece imóvel incorporado em moléculas orgânicas, particularmente na parede celular, o K, soluto inorgânico mais móvel no floema, desde que é absorvido no solo, continua na forma iônica $\mathrm{K}^{+}$, podendo ser redistribuído no interior da planta, provocando diluição em órgãos mais senis (BINKLEY, 1986; MARSCHNER, 1995; EPSTEIN e BLOOM, 2006).

Desconsiderando o $\mathrm{S}$, já que não é determinado em muitos estudos, a sequência de acúmulo $\mathrm{Mg}>\mathrm{P}$ verificada neste estudo é mais frequente em povoamentos adultos (VALERI, 1988; OKI, 2002; BARROS FILHO, 2003 VOIGTLAENDER, 2007). Em plantios mais jovens de Pinus taeda, a posição desses dois nutrientes pode sofrer inversão (BARROS FILHO, 2003 VOIGTLAENDER, 2007). Voigtlaender (2007) estudou o estoque de nutrientes em dois povoamentos de Pinus taeda, com 4 e 8 anos de idade, nas mesmas condições de sítio, onde determinou a seguinte sequência de acú- 
mulo: $\mathrm{N}>\mathrm{K}>\mathrm{Ca}>\mathrm{P}>\mathrm{Mg}$. Barros Filho (2003) relatou a mesma sequência para um povoamento de 2 anos de idade, ao passo que em um povoamento de 4 anos foi a seguinte: $\mathrm{N}>\mathrm{K}>\mathrm{Ca}>\mathrm{Mg}>\mathrm{P}$.

Quanto ao $\mathrm{S}$, as informações são mais escassas, mas esse nutriente está entre os menos abundantes entre os macronutrientes, geralmente com conteúdo semelhante ao $\mathrm{Mg}$, como foi constatado neste estudo (SCHUMACHER et al., 2002; WITSCHORECK, 2008; CALDATO, 2011). Witschoreck (2008) e Caldato (2011) relataram a mesma sequência de acúmulo de nutrientes na biomassa acima do solo: $\mathrm{N}>\mathrm{Ca}>\mathrm{K}>\mathrm{Mg}>\mathrm{S}>\mathrm{P}$, sendo que o conteúdo em $\mathrm{kg} \mathrm{ha}^{-1}$ de $\mathrm{Mg}$ e $\mathrm{S}$ foi de 80,0 e 74,8 e 103,7 e 86,4, respectivamente.

Em relação aos resultados do presente trabalho, alguns dados divergentes foram apresentados por Oki (2002) sobre o estoque de nutrientes, em $\mathrm{kg} \mathrm{ha}^{-1}$, em um povoamento de Pinus taeda de 25 anos de idade: $\mathrm{N}(1004,8), \mathrm{Ca}(408,6), \mathrm{Mg}$ $(371,6), \mathrm{K}(345,7), \mathrm{S}(269,1)$ e P $(23,4)$; o que indica que as características de sítio e manejo podem influenciar os padrões de alocação dos nutrientes.
No caso dos micronutrientes, a sequência de acúmulo obtida neste trabalho foi a mesma apresentada por Caldato (2011) e Oki (2002), respectivamente, para um povoamento de Pinus taeda de 13 e 25 anos de idade; sendo que o último autor não analisou B. Porém, para a mesma espécie em um povoamento de 17 anos de idade Witschoreck (2008) referiu a seguinte sequência de acúmulo (em $\left.\mathrm{kg} \mathrm{ha}^{-1}\right)$ : Fe $(11,8), \mathrm{Mn}(10,5), \mathrm{B}(1,4), \mathrm{Zn}(1,3) \mathrm{e}$ $\mathrm{Cu}(0,6)$.

Em termos relativos, devido à grande diferença no teor de nutrientes entre os componentes das árvores, o padrão de distribuição dos nutrientes não repete a sequência de acúmulo de biomassa (REIS e BARROS, 1990). Os componentes da copa (galhos e acículas), apesar de acumularem apenas $24,3 \%$ da biomassa total, representam $47,7 \%$ do estoque total de nutrientes. Por sua vez, de modo mais marcante, as acículas com apenas 3,4\% da biomassa, acumulam $19,0 \%$ dos nutrientes, com destaque para N, P e K, com $27,5 \%, 25,4 \%$ e $23,6 \%$, respectivamente (Figura 1). Por outro lado, devido à menor concentração de nutrientes em relação aos componentes da

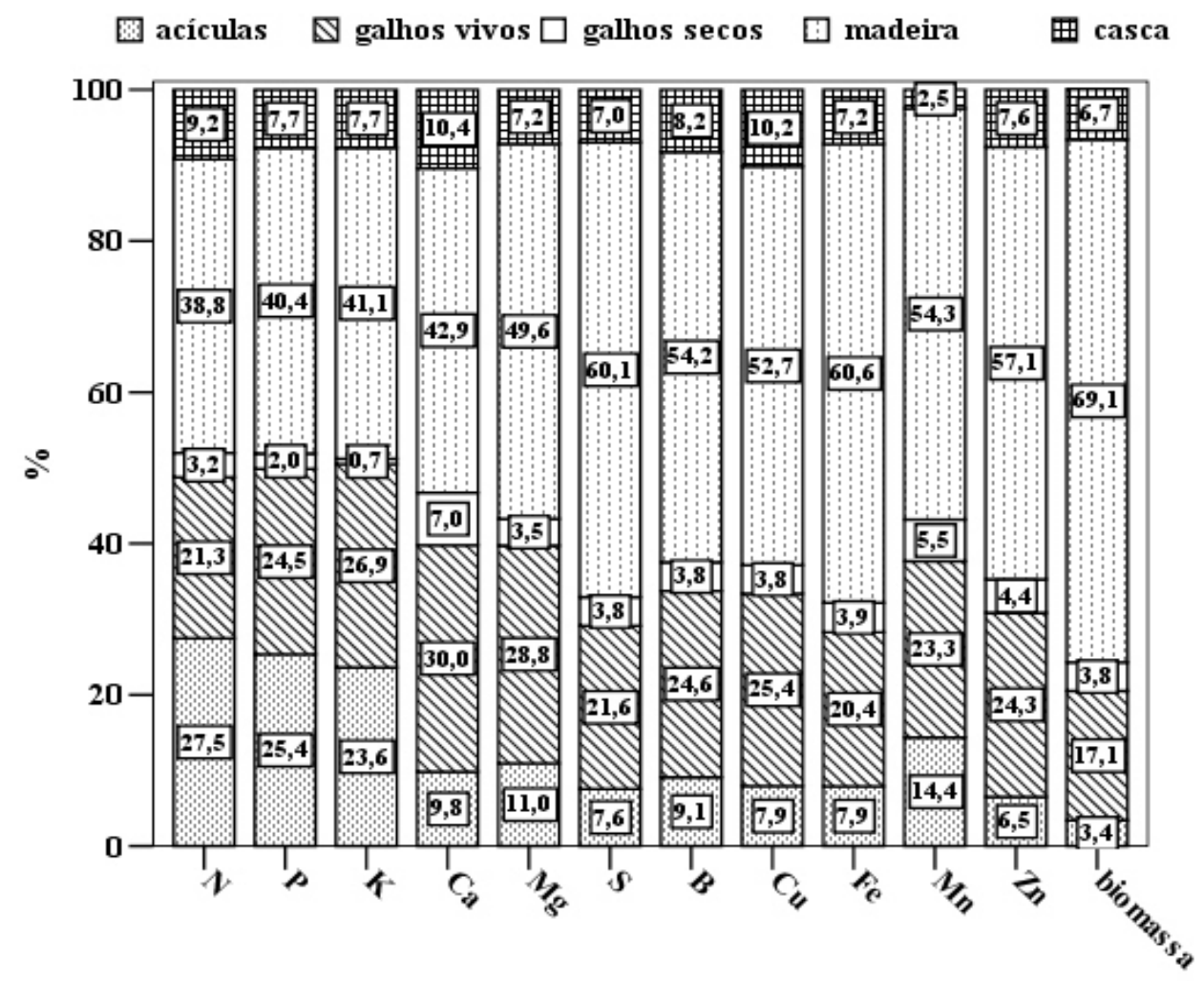

FIGURA 1: Distribuição relativa de biomassa e nutrientes nos diferentes componentes das árvores de Pinus taeda com 27 anos de idade, em Cambará do Sul, RS.

FIGURE 1: Biomass and nutrients relative distribution in different components from 27 year Pinus taeda trees, in Cambará do Sul, RS state. 
copa, a madeira do tronco representou $69,1 \%$ da biomassa total e 43,6 \% do estoque de nutrientes. Essas informações são corroboradas pela literatura, entre outros, Valeri (1988), Oki (2002), Bizon (2005), Voigtlaender (2007), Witschoreck (2008) e Caldato (2011). Segundo Bellote e Silva (2004), a concentração dos nutrientes nos componentes da biomassa está relacionada com suas funções, apresentando, geralmente, o seguinte gradiente: folha $>$ casca $>$ ramo $>$ tronco (alburno $>$ cerne). Ao passo que Marschner (1995) afirma que as folhas (acícula) são o grande centro metabólico da planta, o que justifica o predomínio dos teores mais elevados de nutrientes, enquanto os teores mais baixos estão associados a componentes que têm função mais estrutural ou de condução, como é o caso do lenho e dos galhos (BINKLEY, 1986).

Quanto ao estoque de nutrientes nos galhos secos, evidencia-se o padrão distinto de alguns nutrientes, quanto à mobilidade no tecido vegetal, onde o maior valor relativo é observado para $\mathrm{Ca}$, sabidamente um nutriente imóvel, enquanto o $\mathrm{P}$ e $\mathrm{K}$, facilmente mobilizados ou lixiviados, apresentam os menores valores (Figura 1).

\section{Implicações nutricionais da colheita florestal}

De modo geral a exportação de nutrientes é proporcional à intensidade de remoção da biomassa, no entanto, é preciso considerar o coeficiente de utilização biológico (CUB) que é a taxa de conversão de nutrientes em biomassa (REIS e BARROS, 1990). Este valor demonstra quantas unidades de biomassa é formada por unidade de nutriente, e quanto maior o valor mais eficiente é a conversão dos nutrientes em biomassa (BARROS et al., 1986). Esse índice é apresentado na Tabela 4 que, em termos práticos, do ponto de vista da sustentabilidade nutricional, deve ser o maior possível, com isso, será exportado menos nutriente por unidade de biomassa.

Os valores médios do CUB para os diferentes nutrientes apresentaram a seguinte sequência: $\mathrm{Cu}>\mathrm{B}>\mathrm{Zn}>\mathrm{Fe}>\mathrm{Mn}>\mathrm{P}>\mathrm{K}>\mathrm{Mg}>\mathrm{S}>\mathrm{Ca}$ $>\mathrm{N}$. Para a mesma espécie vegetal e região deste trabalho, Witschoreck (2008) apresentou a seguinte ordem para o $\mathrm{CUB}: \mathrm{Cu}>\mathrm{Zn}>\mathrm{B}>\mathrm{Fe}>\mathrm{Mn}>$ $\mathrm{P}>\mathrm{S}>\mathrm{Mg}>\mathrm{K}>\mathrm{Ca}>\mathrm{N}$. O teor nutricional e a distribuição de biomassa que condicionam o CUB podem variar em função de vários fatores: espécie (capacidade de absorção, distribuição e utilização dos nutrientes); componente da biomassa; solo (disponibilidade de nutrientes); idade da floresta e condições de desenvolvimento (densidade de plantio, competição); entre outros (PEREIRA et al., 1984; WARING e SCHLESINGER, 1985; BINKLEY, 1986; TEIXEIRA et al., 1989; REIS e BARROS, 1990; PRITCHETT, 1990; PALLARDY, 2008).

TABELA 4: Coeficiente de utilização biológico para os componentes da biomassa de Pinus taeda de 27 anos de idade, em Cambará do Sul, RS.

TABLE 4: Biological utilization coefficient for biomass components in 27 year Pinus taeda, Cambará do Sul RS state.

\begin{tabular}{ccccccc}
\hline \multirow{2}{*}{ Nutriente } & \multicolumn{5}{c}{ Componente da biomassa } & \multirow{2}{*}{ Média } \\
\cline { 2 - 6 } & Acículas & Galhos vivos & Galhos secos & Madeira do tronco & Casca do tronco & \\
\hline $\mathrm{N}$ & 64,3 & 417,2 & 604,7 & 924,9 & 377,4 & 477,7 \\
$\mathrm{P}$ & 803,1 & 4180,1 & 10979,4 & 10252,9 & 5200,3 & 6283,2 \\
$\mathrm{~K}$ & 219,7 & 969,9 & 8156,2 & 2565,7 & 1326,6 & 2647,6 \\
$\mathrm{Ca}$ & 296,2 & 488,0 & 463,1 & 1379,9 & 549,7 & 635,4 \\
$\mathrm{Mg}$ & 795,4 & 1520,6 & 2738,9 & 3571,9 & 2391,7 & 2203,7 \\
$\mathrm{~S}$ & 1036,5 & 1827,3 & 2287,1 & 2650,5 & 2212,0 & 2002,7 \\
\hline Média & 535,9 & 1567,2 & 4204,9 & 3557,6 & 2009,6 & 2375,0 \\
$\mathrm{~B}$ & 33806,6 & 62870,5 & 89858,6 & 115460,2 & 73456,0 & 75090,4 \\
$\mathrm{Cu}$ & 184029,9 & 288690,3 & 430076,4 & 563670,7 & 282424,0 & 349778,3 \\
$\mathrm{Fe}$ & 6592,3 & 12870,2 & 14807,8 & 17480,1 & 14266,3 & 13203,4 \\
$\mathrm{Mn}$ & 1714,8 & 5313,6 & 4945,1 & 9217,4 & 19470,0 & 8132,2 \\
$\mathrm{Zn}$ & 31187,3 & 41903,0 & 50681,6 & 72221,6 & 52534,4 & 49705,6 \\
Média & 51466,2 & 82329,5 & 118073,9 & 155610,0 & 88430,1 & 99182,0 \\
\hline
\end{tabular}


Entre os componentes da biomassa, sem exceção, as acículas foram o componente da biomassa que apresentou a menor eficiência de utilização de nutrientes; enquanto a madeira do tronco, exceto para $\mathrm{P}, \mathrm{K}$ e $\mathrm{Cu}$, foi o componente mais eficiente (Tabela 4). Portanto, em valores absolutos, em relação aos outros componentes da biomassa, é através das acículas que, proporcionalmente, exportam-se mais nutrientes por meio da remoção de uma unidade de biomassa, especialmente de N, K e $\mathrm{Ca}$ entre os macronutrientes e $\mathrm{Fe}$ e $\mathrm{Mn}$ entre os micronutrientes. Em relação à madeira do tronco, a remoção de uma unidade de biomassa de acículas acarreta uma exportação de nutrientes, em média, 8,4 vezes superior para os macronutrientes e $3,4 \%$ superior para os micronutrientes, com destaque para N, P, K e Mn, com valores de 14,$4 ; 12,8 ; 11,7$ e 5,4, respectivamente.

Em média, a eficiência de utilização dos nutrientes entre os diferentes componentes da biomassa ficou assim distribuída: galhos secos $>$ madeira do tronco $>$ casca do tronco $>$ galhos vivos $>$ acículas, para os macronutrientes, e madeira do tronco $>$ galhos $\operatorname{secos}>$ casca do tronco $>$ galhos vivos $>$ acículas, para os micronutrientes. Witschoreck (2008) obteve a mesma distribuição para os micronutrientes, distinguindo-se a distribuição para os macronutrientes: madeira do tronco $>$ galhos secos $>$ galhos vivos $>$ casca do tronco $>$ acículas.

A diferenciação na concentração de nutrientes, entre componentes, e dentro de um mesmo componente, tende a se intensificar com a idade, em decorrência da ciclagem interna, que desloca nutrientes de tecidos senescentes para regiões com maior atividade metabólica, causando um processo de diluição dos nutrientes e aumento do CUB (REIS e BARROS, 1990; PALLARDY, 2008). De forma expedita, a simples relação entre os valores do CUB nos galhos vivos e secos indica a capacidade de retranslocação dos nutrientes por meio da ciclagem interna (Tabela 4), o que é diretamente proporcional à mobilidade do nutriente no tecido vegetal (GONÇALVES et al., 2004; REIS e BARROS, 1999). Esse fenômeno é especialmente importante para a manutenção dos nutrientes no interior da planta, e associado a processos de lixiviação e decomposição, pode explicar os maiores valores para $\mathrm{P}$ e $\mathrm{K}$ nos galhos secos (Tabela 4); o que também foi verificado por Witschoreck (2008) nos galhos secos para K e S.

Os elevados valores para o CUB na madei- ra do tronco devem estar relacionados ao processo de diferenciação de alburno em cerne, que ocorre nos tecidos lenhos, promovendo a ciclagem interna de nutrientes e o aumento da eficiência de utilização dos nutrientes (REIS e BARROS, 1990). Por apresentar menor concentração nutricional, e como a proporção de cerne aumenta com a idade do povoamento (WARING e SCHLESINGER, 1985), a exportação de nutrientes com uma unidade de biomassa de madeira do tronco, geralmente, é menor em povoamentos mais velhos.

Para avaliar o impacto nutricional da colheita florestal, na Tabela 5 apresenta-se um comparativo entre diferentes tipos de colheita florestal, envolvendo diferentes intensidades de remoção da biomassa, em termos absolutos e relativos.

A diferença no CUB dos componentes das árvores faz com que o incremento na exportação de nutrientes, em função da intensidade de colheita florestal, não ocorra na mesma ordem de grandeza da remoção de biomassa. A colheita, principalmente dos componentes da copa, que, em média, apresentam CUBs bem mais baixos, quando comparados com os da madeira, pode dobrar a exportação de nutrientes, como verificado para $\mathrm{N}$, $\mathrm{P}, \mathrm{K}$ e Ca (Tabela 5). A colheita da casca, além da madeira, reflete um aumento de $9,7 \%$ na remoção de biomassa, enquanto a exportação de nutrientes acresce entre $4,6 \%$ para o Mn e $24,3 \%$ para o Ca. Caso os componentes da copa (galhos e acículas) também sejam removidos, a elevação na exportação de nutrientes varia de $58,0 \%$ para o $\mathrm{F}$ e de $127,4 \%$ para o $\mathrm{N}$, enquanto a biomassa aumenta apenas $40,8 \%$.

Esses resultados corroboram as informações reportadas por Oki (2002); Bizon (2005); Witschoreck (2008); Caldato (2010), para o incremento na exportação e nutrientes em função da remoção da biomassa de Pinus taeda. Witschoreck (2008) estimou um acréscimo na exportação de nutrientes entre a remoção apenas da madeira do tronco e da árvore inteira de 152,8 para $\mathrm{N}$; 253,1 para $\mathrm{P} ; 107,8$ para $\mathrm{K} ; 98,9$ para $\mathrm{Ca} ; 97,3$ para $\mathrm{Mg} ; 50,6$ para $\mathrm{S} ; 109,0$ para $\mathrm{B} ; 143,5$ para $\mathrm{Cu}$; 522,5 para Fe; 95,7 para $\mathrm{Mn}$ e 160,8 para $\mathrm{Zn}$; enquanto o aumento na remoção de biomassa foi de 40,5 \%. Da mesma forma, Bizon (2005), também verificou aumento na taxa de exportação de nutrientes entre o sistema de colheita somente da madeira do tronco e da árvore inteira para N, P, K, $\mathrm{Ca}$ e $\mathrm{Mg}$, de $117 \%, 147 \%, 95 \%, 81 \%$ e $89 \%$, respectivamente. 
TABELA 5: Exportação de nutrientes $\left(\mathrm{kg} \mathrm{ha}^{-1}\right)$ em função da intensidade de remoção da biomassa de Pinus taeda de 27 anos de idade, em Cambará do Sul, RS.

TABLE 5: Nutrients export $\left(\mathrm{kg} \mathrm{ha}^{-1}\right)$ due to biomass harvesting intensity in 27 year Pinus taeda in Cambará do Sul, RS state.

\begin{tabular}{ccccc}
\hline \multirow{2}{*}{ Variável } & \multicolumn{4}{c}{ Intensidade de colheita da biomassa } \\
\cline { 2 - 5 } & Madeira & Madeira + casca & Madeira + casca + galhos & Árvore inteira \\
\hline $\begin{array}{c}\text { Biomassa } \\
\left(\mathrm{Mg} \mathrm{ha}^{-1}\right)\end{array}$ & 183,83 & $201,60(9,7)$ & $257,04(36,3)$ & $266,08(40,8)$ \\
\hline $\mathrm{N}$ & 198,76 & $245,85(23,7)$ & $371,31(70,2)$ & $511,96(127,4)$ \\
$\mathrm{P}$ & 17,93 & $21,35(19,1)$ & $33,13(71,2)$ & $44,39(123,9)$ \\
$\mathrm{K}$ & 71,65 & $85,05(18,7)$ & $133,13(72,3)$ & $174,26(120,6)$ \\
$\mathrm{Ca}$ & 133,22 & $165,56(24,3)$ & $280,26(88,8)$ & $310,78(107,2)$ \\
$\mathrm{Mg}$ & 51,47 & $58,90(14,4)$ & $92,43(69,5)$ & $103,80(88,8)$ \\
$\mathrm{S}$ & 69,36 & $77,39(11,6)$ & $106,63(48,2)$ & $115,35(59,4)$ \\
\hline $\mathrm{B}$ & 1,59 & $1,83(15,2)$ & $2,67(58,7)$ & $2,94(73,2)$ \\
$\mathrm{Cu}$ & 0,33 & $0,39(19,3)$ & $0,57(62,6)$ & $0,62(75,2)$ \\
$\mathrm{Fe}$ & 10,52 & $11,76(11,8)$ & $15,97(46,4)$ & $17,34(58,0)$ \\
$\mathrm{Mn}$ & 19,94 & $20,86(4,6)$ & $31,43(55,1)$ & $36,70(80,4)$ \\
$\mathrm{Zn}$ & 2,55 & $2,88(13,3)$ & $4,16(56,2)$ & $4,46(66,2)$ \\
\hline
\end{tabular}

Em que: Valores entre parênteses referem-se ao aumento percentual da exportação de biomassa e nutrientes, em relação à colheita apenas da madeira.

A grande representatividade em termos de acúmulo de nutriente nos galhos e acículas, aliado ao caráter mais dinâmico desses nutrientes (ciclagem bioquímica e biogeoquímica), atestam a importância do manejo da biomassa e dos resíduos de colheita desses componentes na sustentabilidade nutricional dos plantios florestais (MILLER, 1995; GONÇALVES et al., 2004; REIS e BARROS, 1999).

Assim, aliada à correta intensidade de colheita da biomassa, a sustentabilidade nutricional, no que diz respeito à manutenção da capacidade produtiva do sítio florestal, deve estar associada ao adequado manejo dos resíduos de colheita. A manutenção dos resíduos distribuídos sobre o solo é uma medida efetiva e recomendável para garantir que os nutrientes presentes nessa biomassa (galhos, acículas e outros componentes), possam retornar ao solo através da ciclagem e suprir parte da demanda nutricional do próximo ciclo da cultura.

\section{CONCLUSÕES}

A biomassa apresentou o seguinte padrão de alocação: madeira $>$ galhos vivos $>$ casca $>$ galhos secos $>$ acículas; enquanto o estoque de nutrientes: madeira $>$ galhos vivos $>$ acículas $>$ casca $>$ galhos secos.
Considerando a biomassa total a sequência de acúmulo de nutrientes foi a seguinte: $\mathrm{N}>\mathrm{Ca}>\mathrm{K}$ $>\mathrm{S}>\mathrm{Mg}>\mathrm{P}>\mathrm{Mn}>\mathrm{Fe}>\mathrm{Zn}>\mathrm{B}>\mathrm{Cu}$.

A remoção de toda a biomassa acima do solo, dependendo do nutriente, aumentou a exportação de nutrientes em até $127,4 \%$, quando comparada com a colheita apenas da madeira do tronco.

A despeito da menor biomassa relativa dos componentes da copa $(24,3 \%)$, a permanência no sítio das acículas ou das acículas e galhos, reduziu a exportação de nutrientes em até $27,5 \%$ e 52,0 \%, respectivamente, em relação à colheita total da biomassa acima do solo.

\section{AGRADECIMENTOS}

À empresa Reflorestadores Unidos S.A. pelo financiamento da pesquisa e apoio logístico nos trabalhos de campo.

\section{REFERÊNCIAS BIBLIOGRÁFICAS}

ALBAUGH, T. J. et al. Long term growth responses of loblolly pine to optimal nutrient and water resource availability. Forest Ecology and Management, Amsterdam, v. 192, n. 1, p. 3-19, Apr. 2004. 
BALDWIN Jr. et al. The effects of spacing and thinning on stand and tree characteristics of 38-year-old loblolly pine. Forest Ecology and Management, Amsterdam, v. 137, n. 1-3, p. 91102, Oct. 2000

BARROS FILHO, N. F. Produção e partição de biomassa e de nutrientes e recomendação de fertilizantes para o Pinus taeda $\mathbf{L}$. (NUTRIPINUS). 2003. 56 f. Dissertação (Mestrado em Engenharia Florestal) - Universidade Federal de Santa Maria, Santa Maria, 2003.

BARROS, N. F. et al. Classificação nutricional de sítios florestais - descrição de uma metodologia. Revista Árvore, Viçosa, v. 10, n. 1, p. 112-120, jan. 1986.

BELlOTE, A. F. J.; SILVA, H. D. Sampling techniques and nutritional evaluations in eucalypt plantations. In: GONÇALVES, J. L. M.; BENEDETTI, V. (eds.) Forest nutrition and fertilization. Piracicaba: IPEF, 2004, cap. 5, p. 113139.

BINKLEY, D. Forest nutrition management. New York: John Wiley \& Sons, 1986. 290 p.

BIZON, J. M. C. Avaliação da sustentabilidade nutricional de plantios de Pinus taeda L. usando um balanço de entrada-saída de nutrientes. 2005. 95 f. Dissertação (Mestrado em Recursos Florestais) - Escola Superior de Agricultura Luiz de Queiroz, Piracicaba, 2005.

CALDATO, S. L. Ciclagem biogeoquímica dos nutrientes em uma plantação de Pinus taeda L. no nordeste argentino. 2011. 106 f. Tese (Doutorado em Engenharia Florestal) - Universidade Federal de Santa Maria, Santa Maria, 2011.

EPSTEIN, E.; BLOOM, A. J. Nutrição mineral de plantas. Londrina, Ed. Planta, 2006. 403 p.

INSTITUTONACIOANALDEMETEOROLOGIA. Oitavo Distrito de Meteorologia ( $8^{\circ}$ DISME). Seção de observação e meteorologia aplicada - SEMA. Porto Alegre, relatório $\mathrm{n}^{\circ}$ 027/2008, protocolado sob n ${ }^{\circ}$ 4294, 24/03/2008, 4 p.

LADEIRA, B. C. et al. Produção de biomassa de eucalipto sob três espaçamentos em uma seqüência de idade. Revista Árvore, Viçosa, v. 25, n. 1, p. 6978, jan./mar. 2001.

LANDSBERG, J. J. Physiological ecology of forest production. London: Academic Press, 1986. $198 \mathrm{p}$.

LEITE, F. P. et al. Crescimento de Eucalyptus grandis em diferentes densidades populacionais. Revista Árvore, Viçosa, v. 21, n. 3, p. 313-321, jul./ set. 1997.
MARSCHNER, H. Mineral nutrition of higher plants. $2^{\text {nd }} e d$. London, Academic Press, 1995. $889 \mathrm{p}$.

MARTIN, T. A.; JOKELA, E. J. Stand development and production dynamics of loblolly pine under a range of cultural treatments in north-central Florida USA. Forest Ecology and Management, Amsterdam, v. 192, p. 39-58, Apr. 2004.

MILLER, H. G. The influence of stand development on nutrient demand, growth and allocation. Plant and Soil, Netherlands, v. 168-169, p. 225-232, 1995.

MIYAZAWA, M.; PAVAN, M. A.; MURAOKA, T. et al. Análises químicas de tecido vegetal. In: SILVA, F.C. (Org.). Manual de análises químicas de solos, plantas e fertilizantes. Brasília: Embrapa Comunicação para Transferência de Tecnologia, 1999. cap. 4, p. 171-224.

MORENO, J. A. Clima do Rio Grande do Sul. Porto Alegre: Secretaria da Agricultura, 1961. 31 p. OKI, V. K. Impactos da colheita de Pinus taeda sobre o balanço hídrico, a qualidade da água e a ciclagem de nutrientes em microbacias. 2002. 71 f. Dissertação (Mestre em Ciências Florestais) - Escola Superior de Agricultura Luiz de Queiroz, Piracicaba, 2002.

PALLARDY, S. Physiology of woody plants. San Diego: Academic Press, 2008. 454 p.

PEREIRA, A. R. et al. Concentração e distribuição de nutrientes em Eucalyptus grandis em função da idade, cultivado na região do cerrado. Brasil Florestal, Brasília, n. 59, p. 27-37, jul./set. 1984.

PRITCHETT, W. L. Suelos forestales: propriedade, conservación y mejoramento. México: Limusa Noriega, 1990. 634 p.

RAMEZOV, N.P. The method of studying the biological cycle of elements in forests. Pochvovedenic, v. 1, p. 71-79, 1959.

REIS, M. G. F.; BARROS, N. F. Ciclagem de nutrientes em plantios de eucalipto. In: BARROS, N. F.; NOVAIS, R. F. (eds). Relação solo eucalipto. Viçosa: Ed. Folha de Viçosa, 1990. cap. 7, p. 265302.

SAS. Statistical analysis system: Programa de computador, ambiente VM. Cary, 1993. Versão 6.08 .

SCHNEIDER, P. R. et al. Análise de regressão aplicada à Engenharia Florestal. 2. ed. Santa Maria: FACOS - UFSM, 2009. 294 p.

SCHUMACHER, M. V. et al. Quantificação da biomassa e do conteúdo de nutrientes no corte raso de uma floresta de Araucaria angustifolia 
(Bert.) O. Ktze. na região de Quedas do Iguaçu - PR. Santa Maria, RS: UFSM/FATECIENS, Departamento de Ciências Florestais. 2002. 78 p. (Relatório Técnico).

SCHUMACHER, M. V. et al. Quantificação da biomassa e nutrientes em um povoamento de Pinus taeda aos 18 anos de idade na região de Cambará do Sul - RS. Santa Maria, RS: UFSM/FATECIENS, Departamento de Ciências Florestais. 2002. 47 p. (Relatório Técnico).

SCHUMACHER, M. V. et al. Quantificação do carbono e dos nutrientes em florestas de eucalipto de diferentes idades. Santa Maria, RS: UFSM/FATECIENS, Departamento de Ciências Florestais, 2003. 112 p. (Relatório Técnico). SCHUMACHER, M. V. et al. Quantificação dos nutrientes no primeiro desbaste de um povoamento de Pinus taeda $L$. no estado do Rio Grande do Sul. Santa Maria, RS: UFSM/FATECIENS, Departamento de Ciências Florestais, 2006. 17 p. (Relatório Técnico).

STRECK, E. V. et al. Solos do Rio Grande do Sul. Porto Alegre: EMATER/RS-ASCAR, 2008. $222 \mathrm{p}$.

SWITZER, G. L.; NELSON, L. E. Nutrient accumulation and cycling in loblolly pine (Pinus taeda L.) plantation ecosystems: the first twenty years. Soil Science Society of America, Madison, v. 36, p. 143-147, Jan./Mar. 1972.

TEIXEIRA, J. L.; BARROS, N. F.; COSTA, L. M. et al. Biomassa e conteúdo de nutrientes de duas espécies de eucalipto em diferentes ambientes do Médio Rio Doce, MG. Revista Árvore, Viçosa, v. 13, n. 1, p. 34-50, jan./jun. 1989.

VALERI, S. V. Exportação de biomassa e nutrientes de povoamentos de Pinus taeda $\mathrm{L}$. desbastados em diferentes idades. 1988. 164 f. Tese (Doutorado em Engenharia Florestal) Universidade Federal do Paraná, Curitiba, 1988.

VOIGTLAENDER, M. Caracterização hidrológica e biogeoquímica de microbacias: uma comparação entre Mata Atlântica e Pinus taeda L. 2007. 74 f. Dissertação (Mestrado em Recursos Florestais) - Escola Superior de Agricultura Luiz de Queiroz, Piracicaba, 2007.

WARING, R. H.; SCHLESINGER, W. H. Forest ecosystems: concepts and management. San Diego: Academic Press, 1985. 340 p.

WITSCHORECK, R. Biomassa e nutrientes no corte raso de um povoamento de Pinus taeda $\mathrm{L}$. de 17 anos de idade no município de Cambará do Sul - RS. 2008. 80 f. Dissertação (Mestrado e Engenharia Florestal) - Universidade Federal de Santa Maria, Santa Maria, 2008.

ZHANG, Y.; BORDERS, B. E. Using a system mixed-effects modeling method to estimate tree compartment biomass for intensively managed loblolly pines an allometric approach. Forest Ecology and Management, Amsterdam, v. 194, n. 1-3, p. 145-157, 14 June 2004. 